\title{
Indirect Bacterial Effect Enhanced Less Recovery of Neonicotinoids by Improved Activities of White-Rot Fungus Phlebia brevispora
}

\author{
Joy L. Harry-Asobara ${ }^{1}$ and Ichiro Kamei ${ }^{2 *}$ \\ ${ }^{1}$ Graduate School of Agriculture and Engineering, University of Miyazaki, 1-1, Gakuen-kibanadai-nishi, Miyazaki 889-2192, Japan \\ ${ }^{2}$ Faculty of Agriculture, University of Miyazaki, 1-1, Gakuen-kibanadai-nishi, Miyazaki 889-2192, Japan
}

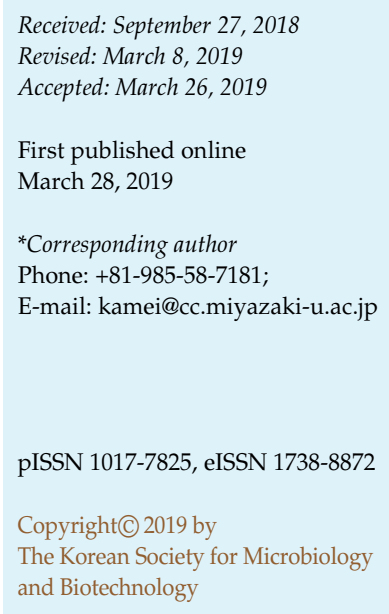

\begin{abstract}
Bacterial strains that improve mycelial morphology and growth of white-rot fungi in liquid medium could enhance the impact of white-rot fungi towards lesser recovery of neonicotinoids when cocultured. This was demonstrated by the recovery of clothianidin and acetamiprid from cocultures of the white-rot fungus Phlebia brevispora strains with two mycelial-growth-promoting bacteria, Enterobacter sp. TN3W-14 and Pseudomonas sp. TN3W-8. Clothianidin recovery from cocultures of white-rot fungi and bacteria was over $40 \%$ lower than that from axenic microbial cultures and mixed-bacterial cultures. About $20 \%$ less acetamiprid was equally recovered from both TMIC33929+TN3W-14 cocultures and mixedbacterial cultures than from axenic fungal and bacterial cultures.
\end{abstract}

Keywords: Bioremediation, fungal remediation enhancement, insecticides, growth-promoting bacteria, white-rot fungi
Neonicotinoids, which are the most widely used insecticides in the world, affect the central nervous system of insects, resulting in paralysis and death [1]. These compounds invariably cause less toxicity to birds and mammals than insects; however, they have been implicated in the death of beneficial insects such as honey bees [2]. Because some neonicotinoids equally degrade slowly and can remain in the environment for months or even years after application [1], there is a need for more proactive degradation and detoxification measures.

Fungi and bacteria have been shown to possess the ability to convert pesticides into simpler, non-toxic compounds. Moreover, white-rot fungi, which grow naturally on wood, are characterized by their ability to decompose lignin and other organic substances [3]. Recent research has focused on developing microbial consortia for complete degradation of such pollutants. This is because microbial consortia consisting of various bacteria and fungi have been found to have superior biodegradation performance than single microbial strains [4].

Our study [5] revealed that two bacterial strains, TN3W8 and TN3W-14, which showed high homology with Pseudomonas and Enterobacter, respectively, were able to improve mycelia growth of the white-rot fungi Phlebia brevispora in liquid medium via dissociation of mycelial gathering. The white-rot fungus $P$. brevispora, which is one of the most studied species, has the ability to degrade organohalogen compounds such as polychlorinated biphenyls, polychlorinated dioxins, and organohalogen pesticides [6-8]. With the hypothesis that this growth enhancement would translate to improved bioremediation traits in white-rot fungus, the present study was conducted to evaluate the impact of $P$. brevispora with or without coculturing with growth-promoting bacteria on recovery of two neonicotinoid insecticides, clothianidin and acetamiprid.

The white-rot fungi $P$. brevispora strains TN3F and TMIC33929, and its growth-promoting bacteria Enterobacter sp. TN3W-14 or Pseudomonas sp. TN3W-8 were incubated on PDB or PDA medium (Difco, France) according to our previous study [5].

Growth media were prepared by adding two 2-cmdiameter mycelial disks to fungal cultures in autoclaved 100-ml Erlenmeyer flasks containing Kirk's low nitrogen medium (LNM) [9] at different culture formulations. Fungal axenic cultures (TMIC33929 or TN3F) contained 10 m1 LNM, while bacterial axenic cultures (TN3W-8 or 
TN3W-14) and fungal-single bacterial (TMIC33929+TN3W8; TMIC33929+TN3W-14 or TN3F+TN3W-8; TN3F+TN3W14) cocultures contained $8 \mathrm{ml} \mathrm{LNM}$ and $2 \mathrm{ml}$ of corresponding preincubated bacterial cells, with an initial cell population of approximately $10^{6}$ cells $\mathrm{ml}^{-1}$. For fungalmixed bacterial cocultures (TMIC33929+TN3W-8+TN3W14 or TN3F+TN3W-8+TN3W-14) and mixed-bacterial cultures (TN3W-8+TN3W-14), $1 \mathrm{ml}$ each of the preincubated bacterial cultures was added to $8 \mathrm{ml}$ of LNM. PDB medium from $0.0 \mathrm{ml}$ to $2.0 \mathrm{ml}$ was added to fungal cultures, in place of bacterial cells, to evaluate its effect on fungal activity. Cultures were then pre-incubated for 5 days on a rotary shaker at $120 \mathrm{rpm}$, after which $200 \mu \mathrm{l}$ of $10 \mathrm{mM}$ substrate (final conc. $0.05 \mathrm{mM}$ ) in $N, N$-dimethylformamide was added. The headspace of each flask was subsequently aseptically flushed with oxygen for $5 \mathrm{~s}$ at inoculation, sealed with a glass stopper and sealing tape, and incubated in the dark on a rotary shaker at $25^{\circ} \mathrm{C}$ for 15 days. For this experiment, abiotic cultures served as a control

At 15 days after sampling, triplicate samples of each culture were homogenized with $10 \mathrm{ml}$ of acetonitrile after addition of internal standard (coumarine, final conc. $0.01 \mathrm{mM}$ ). To quantify the amount of substrate recovery, the resulting supernatants were analyzed by high performance liquid chromatography (HPLC). HPLC was performed on a Shimadzu LC-10AD with a SPD-20A UV/VIS (254 nm) detector and a STR ODS-II $250 \times 4.6 \mathrm{~mm}$ column. T-tests were used to determine the level of significance at a $p$ value of 0.01 for clothianidin and 0.05 for acetamiprid. To determine the stability of the reactants, half-lives and rate constants of the first order reaction were calculated using the first order degradation rate model [10] as described below:

$$
X_{\mathrm{t}} / 2=\mathrm{X}_{0} \mathrm{e}^{-\mathrm{kt} 1 / 2},
$$

where $X_{t}$ was the concentration of neonicotinoid insecticides $(\mu \mathrm{M})$ at time $\mathrm{t}$ (days), $\mathrm{k}$ was a first order degradation constant $\left(d^{-1}\right)$ and $t_{1 / 2}$ was the half-life. By applying nonlinear regression to the experimental data, $k=0.693 / t_{1 / 2}$ was obtained.

To determine metabolites, the 10-ml contents from each flask were adjusted to $\mathrm{pH} 2.5$ then transferred to separating funnels and extracted twice with an equal volume of ethyl acetate. The ethyl acetate phase was pooled and dried by a rotary evaporator and nitrogen gas. The dried samples were subsequently dissolved in methanol $(600 \mu \mathrm{l})$ for HPLC analysis.

In the present study, axenic fungal and bacterial cultures gave high recovery of both clothianidin and acetamiprid. For clothianidin (Table 1), cultures of fungal strains TN3F and TMIC33929 recovered $68.9 \%$ and $87.7 \%$ of substrate at estimated half-lives of 29.7 days $(\mathrm{k}=0.023)$ and 37.8 days $(\mathrm{k}=0.018)$ respectively, which were significantly higher $(p>0.01)$ than the recovery from the coculture samples, while cultures of bacterial strains TN3W-14 and TN3W-8 recovered almost all substrate added, similar to the abiotic control. Fungal cocultures with each of the bacterial strains recovered about $30 \%$ of clothianidin after 15 days of incubation. TMIC33929+TN3W-14 and TMIC33929+TN3W8 cocultures recovered $22.9 \%$ and $24.2 \%$ of the substrate at an estimated half-life of 10 days $(\mathrm{k}=0.070$ and 0.066$)$ respectively, while $\mathrm{TN} 3 \mathrm{~F}+\mathrm{TN} 3 \mathrm{~W}-14$ and $\mathrm{TN} 3 \mathrm{~F}+\mathrm{TN} 3 \mathrm{~W}-8$ cocultures recovered $31.3 \%$ and $26.4 \%$ of the clothianidin at estimated half-lives of 13.5 days $(k=0.051)$ and 11.4 days $(\mathrm{k}=0.061)$ respectively. Substrate recovery from mixedbacterial cultures was also about $50 \%$ higher than that from fungal-bacterial cocultures. The half-life of clothianidin has been noted to range from 148-1155 days [11], although half-life and behavior of clothianidin are governed by a variety of complex dynamic physical, chemical, and

Table 1. Clothianidin recovery $R(\%)$, half-life $t_{1 / 2}(d)$ and rate constant $k\left(d^{-1}\right)$ for fungal axenic cultures and cocultures with bacterial strains.

\begin{tabular}{|c|c|c|c|c|c|c|c|c|c|c|c|}
\hline & \multicolumn{11}{|c|}{ Fungal and bacterial combinations } \\
\hline Fungi & TN3F & TN3F & TN3F & TN3F & ТМIC33929 & TMIC33939 & TMIC3393 & TMIC33929 & & & \\
\hline Bacteria & & TN3W-8 & TN3W-14 & $\begin{array}{c}\text { TN3W-8 } \\
\text { TN3W-14 }\end{array}$ & & TN3W-8 & TN3W-14 & $\begin{array}{l}\text { TN3W-8 } \\
\text { TN3W-14 }\end{array}$ & TN3W-8 & TN3W-14 & $\begin{array}{c}\text { TN3W-8 } \\
\text { TN3W-14 }\end{array}$ \\
\hline $\mathrm{R}(\%)$ & 68.9 & $26.4^{*}$ & $31.3^{*}$ & $17.2^{*}$ & 87.7 & $24.2^{*}$ & $22.9^{*}$ & $15.1^{*}$ & 109.4 & 96.7 & 76.8 \\
\hline & \pm 2.4 & \pm 3.5 & \pm 2.1 & \pm 4.9 & \pm 11.6 & \pm 1.8 & \pm 1.1 & \pm 1.8 & \pm 15.4 & \pm 4.3 & \pm 1.8 \\
\hline$t_{1 / 2}(d)$ & 29.7 & 11.4 & 13.5 & 7.4 & 37.8 & 10.4 & 9.9 & 6.5 & 47.1 & 41.7 & 33.1 \\
\hline $\mathrm{k}\left(\mathrm{d}^{-1}\right)$ & 0.023 & 0.061 & 0.051 & 0.094 & 0.018 & 0.066 & 0.070 & 0.107 & 0.015 & 0.017 & 0.021 \\
\hline
\end{tabular}

Recovery values are means \pm standard deviations of triplicate samples.

*Asterisks represent coculture recovery values, which are significantly different from corresponding fungal axenic cultures at $p>0.01$. Recovery from abiotic control was $99.8 \pm 5.9$. 
Table 2. Acetamiprid recovery $\mathrm{R}(\%)$, half-life $\mathrm{t}_{1 / 2}(\mathrm{~d})$ and rate constant $\mathrm{k}\left(\mathrm{d}^{-1}\right)$ for axenic cultures of Phlebia brevispora TMIC33929, cultures of bacterial strains TN3W-14 and TN3W-8 and fungal-bacterial cocultures.

\begin{tabular}{|c|c|c|c|c|c|c|c|}
\hline \multirow[b]{2}{*}{ Fungi } & \multicolumn{7}{|c|}{ Fungal and bacterial combinations } \\
\hline & TMIC33929 & TMIC33939 & TMIC3393 & TMIC33929 & & & \\
\hline Bacteria & & $+\mathrm{TN} 3 \mathrm{~W}-8$ & +TN3W-14 & $\begin{array}{c}+\mathrm{TN} 3 \mathrm{~W}-8 \\
+\mathrm{TN} 3 \mathrm{~W}-14\end{array}$ & TN3W-8 & TN3W-14 & $\begin{array}{c}\text { TN3W-8 } \\
+\mathrm{TN} 3 \mathrm{~W}-14\end{array}$ \\
\hline \multirow[t]{2}{*}{$\mathrm{R}(\%)$} & 81.59 & 76.88 & $63.37^{*}$ & $64.63^{*}$ & 103.90 & 78.64 & 63.35 \\
\hline & \pm 2.6 & \pm 12.3 & \pm 2.5 & \pm 3.6 & \pm 1.8 & \pm 4.7 & \pm 8.9 \\
\hline $\mathrm{k}\left(\mathrm{d}^{-1}\right)$ & 0.020 & 0.021 & 0.025 & 0.025 & 0.015 & 0.020 & 0.025 \\
\hline
\end{tabular}

Recovery values are means \pm standard deviations of triplicate samples.

*Asterisks represent coculture recovery values, which are significantly different from corresponding fungal axenic cultures at $p>0.05$. Recovery from abiotic control was $101.1 \pm 0.3$.

biological processes [12]. Few studies have however achieved shorter half-lives in non-soil conditions [13, 14], especially with enriched microbial cultures. In the present study, shorter half-lives of clothianidin in fungal-bacterial cocultures along with clear transformation of the substrate were observed, representing strong points of this less recovery strategy. Similarly for acetamiprid (Table 2), coculture of TMIC33929+TN3W-14 recovered $63.3 \%$ of the substrate at an estimated half-life of 27.3 days $(\mathrm{k}=0.025)$, which was significantly lower $(p>0.05)$ than the $81.5 \%$ recovery observed for TMIC33929 cultures at an estimated half-life of 35.1 days $(k=0.020)$. Acetamiprid has generally been noted to degrade more slowly in water than in soils $[15,16]$.

To avoid the concerns about the effect of PDB addition on fungal activity, PDB medium without bacterial inoculation was added to the LNM with $P$. brevispora. The results showed that the addition of little or no PDB gave relatively the same quantity of insecticide recovery as axenic fungal cultures, while the addition of higher levels of PDB tended to increase substrate recovery (data not shown). Few studies have shown that bacterial strains with little or no degradative activity for an insecticide could enhance fungal reduction of the same insecticide. Nevertheless, the present study revealed a remarkable increase in the mycelial growth of coculture samples, along with less recovery of neonicotinoids by improved basidiomycetes' activities.

Possible metabolic products from fungal and bacterial treated cultures of acetamiprid were found in ethyl acetate extracts by HPLC analysis (Fig. 1). A peak corresponding to the compound, I, appeared on the axenic TMIC33929 chromatogram at a retention time of $2.74 \mathrm{~min}$, while another peak, II, appeared on chromatograms of TN3W-
$14+\mathrm{TN} 3 \mathrm{~W}-8$ coculture at a retention time of $3.41 \mathrm{~min}$ Furthermore, detection of compound III at $4.36 \mathrm{~min}$ from fungal-bacterial cocultures alone suggests cooperative degradation by the fungus and the bacterium. Although further detailed study should be needed to identify these compounds, these detected compounds did not appear on chromatograms of biotic control samples.

This paper reported the indirect use of bacterial strains (of low insecticide metabolism) in achieving enhanced fungal activity for lesser insecticide recovery, by establishing a
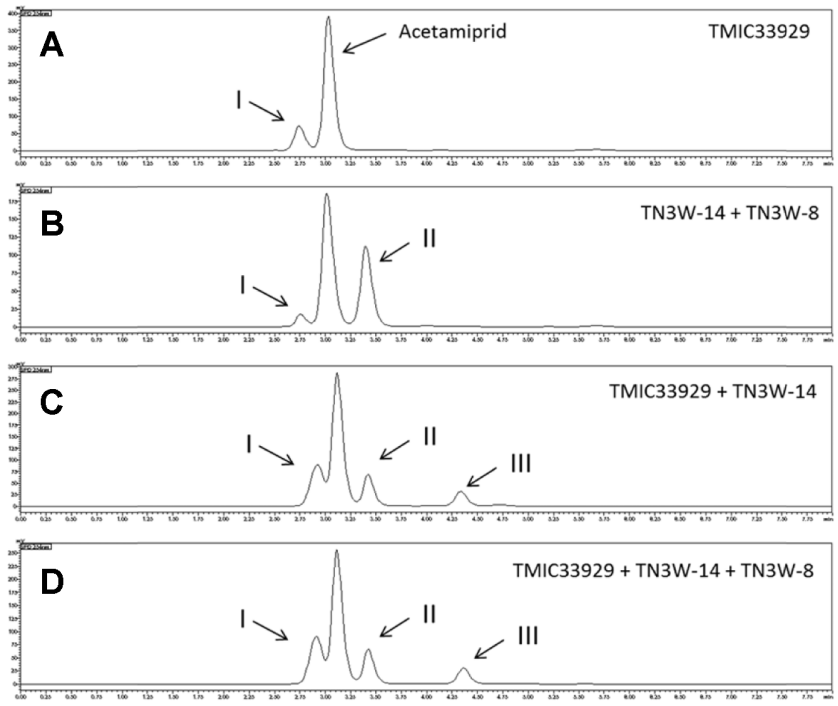

Fig. 1. Possible metabolic compounds during acetamiprid recovery in Kirk low nitrogen medium.

Products in acetamiprid culture with fungal strain TMIC33929 (A), mixed bacterial coculture of strains TN3W-14 and TN3W-8 (B), fungal-bacterial coculture of fungal strain TMIC33929 with bacterial strain TN3W-14 (C), and fungal-mixed bacterial coculture of fungal strain TMIC33929 with bacterial strains TN3W-14 and TN3W-8 (D). 
mycelial morphological and growth-promoting relationship between basidiomycetes and bacteria in liquid medium.

\section{Acknowledgments}

The authors are grateful for the support of this research by a Grant-in-Aid for Scientific Research from the Ministry of Education, Culture, Sports, Science and Technology of Japan (grant nos. 23688041 and 15K14905). We thank Jeremy Kamen, MSc, from Edanz Group (www.edanzediting.com/ ac) for editing a draft of this manuscript.

\section{Conflict of Interest}

The authors have no financial conflicts of interest to declare.

\section{References}

1. Goulson D. 2013. An evaluation of the environmental risks posed by neonicotinoid insecticides. J. Appl. Ecol. 50: 977-987.

2. Daily News. 2017. Strongest evidence yet that neonicotinoids are killing bees. Available from https://www.newscientist. com/article/2139197-strongest-evidence-yet-that-neonicotinoidsare-killing-bees/. Accessed May 4, 2018.

3. Buswell JA. 1991. Fungal degradation of lignin, pp. 425-480. In Arora K, Mukerij KG, Knudsen G (eds.), Handbook of applied mycology, Marcel Dekker, New York,

4. Boonchan S, Britz ML, Stanley G.A. 2000. Degradation and mineralization of high-molecular-weight polycyclic aromatic hydrocarbons by defined fungal-bacterial cocultures. Appl. Environ. Microbiol. 66: 1007-1019.

5. Harry-asobara JL, Kamei I. 2018. Bacterial strains isolated from Cedar wood improve the mycelial growth and morphology of white rot fungus Phlebia brevispora on agar and liquid medium. J. Wood Sci. 64: 444- 450.
6. Kamei I, Suhara H, Kondo R. 2005. Phylogenetical approach to isolation of white-rot fungus capable of degrading polychlorinated dibenzo-p-dioxin. Appl. Microbiol. Biotechnol. 69: 358-366.

7. Kamei I, Sonoki S, Haraguchi K, Kondo R. 2006. Fungal bioconversion of toxic polychlorinated biphenyls by whiterot fungus P. brevispora. Appl. Microbiol. Biotechnol. 73: 932940.

8. Xiao P, Mori T, Kamei I, Kondo R. 2011. Metabolism of organochlorine pesticide heptachlor and its metabolite heptachlor epoxide by white rot fungi belonging to genus Phlebia. FEMS Microbiol. Lett. 314: 140-146.

9. Kirk TK, Yang HH, Keyser P. 1978. The chemistry and physiology of the fungal degradation of lignin. Dev. Ind. Microbiol. 19: 51-61.

10. First order reactions. Chemistry Libre Texts. 2017. Available from https://chem.libretexts.org/Core/Physical_and_Theoretical_ Chemistry/Kinetics/Reaction_Rates/First-Order_Reactions. Accessed Jan. 25, 2018.

11. Clothianidin- Source Watch. https://www.sourcewatch.org/ index.php/Clothianidin. Accessed Feb. 22, 2019.

12. Fang XH, Qiu RL. 2002. Research of the behavior of pesticide in soil environment. Soil Environ. Sci. 11: 94-97.

13. Li L, Jiang G, Liu C, Liang H, Sun D, Li W. 2012. Clothianidin dissipation in tomato and soil, and distribution in tomato peel and flesh. Food Control 25: 265-269.

14. Lawrence IG, Sarjeet SG. 2010. The neonicotinoid insecticides, pp. 89-90. In: Insect control: biological and synthetic agents. Science, Academic Press.

15. Wang G, Yue W, Liu Y, Li F, XiongM ZH. 2013a. Biodegradation of the neonicotinoid insecticide Acetamiprid by bacterium Pigmentiphaga sp. strain AAP-1 isolated from soil. Bioresour. Technol. 138: 359-368.

16. Krull R, Wucherpfennig $T$, Esfandabadi ME, Walisko R, Melzer G, Hempel DC, et al. 2013. Characterization and control of fungal morphology for improved production performance in biotechnology. J. Biotechnol. 163: 112-123. 